\title{
Visual and Somatosensory Information about Object Shape Control Manipulative Fingertip Forces
}

\author{
Per Jenmalm and Roland S. Johansson \\ Department of Physiology, Umeå University, S-901 87 Umeå, Sweden
}

We investigated the importance of visual versus somatosensory information for the adaptation of the fingertip forces to object shape when humans used the tips of the right index finger and thumb to lift a test object. The angle of the two flat grip surfaces in relation to the vertical plane was changed between trials from -40 to $30^{\circ}$. At $0^{\circ}$ the two surfaces were parallel, and at positive and negative angles the object tapered upward and downward, respectively. Subjects automatically adapted the balance between the horizontal grip force and the vertical lift force to the object shape and thereby maintained a rather constant safety margin against frictional slips, despite the huge variation in finger force requirements. Subjects used visual cues to adapt force to object shape parametrically in anticipation of the force requirements imposed once the object was contacted. In the absence of somatosensory information from the digits, sighted subjects still adapted the force coordination to object shape, but without vision and somatosensory inputs the performance was severely impaired. With normal digital sensibility, subjects adapted the force coordination to object shape even without vision. Shape cues obtained by somatosensory mechanisms were expressed in the motor output about $0.1 \mathrm{sec}$ after contact. Before this point in time, memory of force coordination used in the previous trial controlled the force output. We conclude that both visual and somatosensory inputs can be used in conjunction with sensorimotor memories to adapt the force output to object shape automatically for grasp stability.

Key words: cutaneous sensibility; digits; fingertip forces; grasp stability; hand; human; intrinsic object properties; manipulation; object shape; somatosensory information; vision
When people lift and hold an object with parallel vertical grip surfaces, they automatically change the horizontal grip forces in parallel with the changes of the vertical lift forces (Johansson and Westling, 1984a; Westling and Johansson, 1984). This parallel increase and decrease in the forces normal and tangential to the grip surfaces represents a constraint used by the nervous system to coordinate the fingertip force vector for grasp stability during a variety of grasp configurations and manipulative tasks (e.g., Johansson and Westling, 1988a,b; Flanagan and Wing, 1993; Flanagan and Tresilian, 1994; Flanagan et al., 1995; Kinoshita et al., 1996). That is, by this constraint we apply adequately large normal forces in relation to destabilizing tangential forces to prevent slips and accidental loss of the object. At the same time, excessive normal (grip) forces are avoided that may cause unnecessary fatigue and may crush fragile objects or injure the hand. Accordingly, we automatically adjust the ratio between the normal and tangential forces to the frictional status at the digit-object interface such that an adequate safety margin against frictional slips is maintained during different frictional conditions (Johansson and Westling, 1984a; Westling and Johansson, 1984; Edin et al., 1992; Cole and Johansson, 1993; Flanagan and Wing, 1995; Forssberg et al., 1995; Cadoret and Smith, 1996). A sensorimotor memory related to the frictional experiences in previous interactions with the object determines the force balance used (Johansson and Westling, 1984a; Edin et al., 1992) according to an "anticipatory parameter control” policy (for an overview, see Johansson, 1996).

Received Dec. 19, 1996; revised March 17, 1997; accepted March 21, 1997.

This study was supported by the Swedish Medical Research Council (project 08667), Department of Naval Research (Arlington, VA) Grant N00014-92-J-1919), and the Göran Gustafsson Foundation for Research in Natural Sciences and Medicine.

Correspondence should be addressed to Per Jenmalm at the above address.

Copyright (C) 1997 Society for Neuroscience $0270-6474 / 97 / 174486-14 \$ 05.00 / 0$
When necessary, however, this memory is updated to frictional changes based on tactile afferent information (Johansson and Westling, 1984a, 1987). This takes place intermittently according to a policy we have termed "discrete event, sensory driven control" (Johansson, 1996). Likewise, subjects also use memory mechanisms to adjust the motor commands parametrically in anticipation of the weight of the object (Johansson and Westling, 1988a), and tactile mechanisms may be used to update this weightrelated memory (Westling and Johansson, 1987).

In addition to sensorimotor memories and tactile information during actual manipulation, visuomotor mechanisms are important in the control of prehensile tasks. When we reach for and grasp objects based on visual cues, we transport the hand toward the target object and preshape and orient the hand to facilitate the act of gripping the object (Jeannerod, 1984; Kelso et al., 1994; Desmurget et al., 1996). Furthermore, the way we position the digits onto the surfaces of an object promotes grasp stability (Goodale et al., 1994b; Flanagan and Wing, 1995) and achievement of further action goals (Rosenbaum and Jorgensen, 1992). Importantly, the kinematics of these movements may be determined largely by the initial view of the object before the movement onset, again indicating the importance of implicit memory control of relevant motor program parameters in prehension (Jackson et al., 1995; Gentilucci et al., 1996).

Visuomotor mechanisms are also involved in anticipatory control of the forces applied to the object, once contacted. That is, the development of the finger forces may be determined initially by a process involving visual identification of the object and the retrieval of implicit memory information concerning its physical properties in terms of the forces to apply. So far, this has been shown to apply to the adaptation of the force output to the 
weights of objects when we lift objects of different weights, sizes, and densities (Gordon et al., 1991, 1993).

The weights of objects and the friction at the hand-object interfaces are not the only intrinsic object features that have to be accommodated in the control of grasp stability. The shape of the object also must be taken into account, because the geometric relationship between the grasp surfaces imposes various constraints on the force coordination (Blake, 1992). First, for each grasp surface the direction of the applied fingertip force must be within the limits imposed by the frictional condition, i.e., within a certain angle relative to the normal of the grasp surface. Second, for equilibrium in any static grasp, all forces and moments applied to the object must sum to zero. In the current study we sought to examine the relative importance of visual versus digital afferent information for the adaptation of the fingertip forces to object shape while subjects lifted objects by using a precision grip.

\section{MATERIALS AND METHODS}

Subjects and general procedure. Experiments were performed on 17 healthy, right-handed subjects ( 6 female and 10 male) ranging in age from 21 to $30 \mathrm{yr}$. All gave their informed consent, and the experimental protocol was approved by the local ethics committee. The subjects were not informed about the specific purpose of the experiments. About 5 min before the experiment they washed their hands with soap and water. During the experiments the subjects sat in a chair with their right upper arm parallel to the trunk and the forearm extending anteriorly. They were asked to use the tips of the right thumb and index finger to lift a test object to a position about $5 \mathrm{~cm}$ above its support. The object was resting on a table top about $5 \mathrm{~cm}$ in front of the hand. After it was grasped the lifting movement took place mainly with elbow flexion.

Test object. The weight of the test object was $690 \mathrm{gm}$, and its center of gravity was located $110 \mathrm{~mm}$ below the center of its two flat grip surfaces $(35 \times 40 \mathrm{~mm})$. These were located on opposite sides of the object and covered by fine grain sandpaper (no. 320). For each grip surface its angle in relation to the vertical plane could be changed in $10^{\circ}$ steps from -40 to $30^{\circ}$. At $0^{\circ}$ the grip surfaces were parallel in two vertical planes spaced $52 \mathrm{~mm}$ apart. In each individual trial the two surfaces were always set at the same angle, and the distance between their centers was kept at 52 $\mathrm{mm}$. At positive and negative angles the object tapered upward and downward, respectively (Fig. $1 A$ ).

The vertical and horizontal forces applied to the object (Fig. $1 B$ ) by each digit were registered continuously, using a multiple strain gauge transducer system $(\mathrm{DC}-100 \mathrm{~Hz})$. The crosstalk between the horizontal and vertical force measurements was $<5 \%$ over the whole grip surfaces. The vertical position of the object was measured with an ultrasonic position transducer $(\mathrm{DC}-100 \mathrm{~Hz})$, the transmitter of which was located in the top of the object and the receiver of which was in the ceiling.

Lift series. Subjects performed two different series of lifts, and between each lift there was a short break lasting for about $5 \mathrm{sec}$. Before the lifting series, the task was demonstrated by the experimenter. Seven sighted subjects performed the first series. It included 40 lifts divided into eight blocks consisting of five consecutive trials, during which the surface angle was kept constant. The shape of the object was changed between blocks in an unpredictable way using the following eight angles: $-40,-30,-20$, $-10,0,10,20$, and $30^{\circ}$; with a $40^{\circ}$ angle, subjects had difficulties lifting the object because of the limits imposed by the friction between the skin and the grip surfaces. The lifts were all terminated by the subject slowly decreasing the grip strength when the object was held in air until it was dropped (Fig. 1C). A sound signal (brief tone) that occurred $5 \mathrm{sec}$ after the object was initially touched instructed the subject when to start to decrease the grip strength.

In the second series, including 82 lifts, we varied unpredictably the surface angle between succeeding lifts using angles of $-30,0$, and $30^{\circ}$. Furthermore, to analyze influences of surface angle in previous trials, each of these angles was preceded nine times by lifts with $-30,0$, and $30^{\circ}$ angles. In this test series the subjects lifted the object as in the first series, but after the auditory signal they replaced the object on the table in an ordinary manner (Fig. $1 D$ ). Ten subjects, different from those participating in the first series, carried out this series with and without vision $(82 \times$ $10 \times 2=1640$ trials). To blindfold the subjects, we covered their eyes with a cloth, and the subjects were instructed to lift the object as they would have done with vision. However, because the blindfolded subjects could not always appropriately orient their hands to the object, the experimenter guided their right hands to a starting position suitable to grasp the object. Four of these subjects repeated the same series during local anesthesia of the index finger and thumb $(82 \times 4 \times 2=656$ trials $)$. The digital nerves were blocked by equal parts of $5 \%$ solutions of bupivacain (Marcain) and prilocain (Citanest). The anesthesia was infiltrated near the digital nerves at the midlevel of the proximal phalanges (about $5 \mathrm{ml} /$ digit) and was considered successful when the subject failed to feel a light touch, as tested with calibrated von Frey hairs (Johansson et al., 1980), a pinprick, and heavy pressure. The stability of the anesthesia was verified after each test series.

If the test object was accidentally dropped as a result of frictional slips, the test series was resumed by repeating the current trial.

Data analysis. Using a custom-built data acquisition and analysis system (SC/ZOOM; Department of Physiology, Umeå University) on a DOS operated 486 system, the transducer signals were sampled at $400 / \mathrm{sec}$ with 12 bit resolution and stored on a computer disk. Forces normal (NF) and tangential (TF) to the grip surface (Fig. $1 B$ ) were computed for each digit from the measured vertical forces (VFs) and horizontal forces (HFs) and the known surface angle $(\alpha)$ between the grip surface and the vertical of the object using the following equations: $N F=H F \times \cos (\alpha)-V F \times$ $\sin (\alpha)$, and $T F=H F \times \sin (\alpha)+V F \times \cos (\alpha)$.

The horizontal, vertical, normal, and tangential forces reported refer to the means of the corresponding forces at the two grip surfaces. Force rates were computed as the first time derivatives of the force signals using a \pm 5 point numerical differentiation, i.e., calculated within windows of $\pm 12.5 \mathrm{msec}$.

The friction between the grip surface and the digits was estimated for each trial in the series with blocked trials by computing the ratio between the normal and tangential forces at the onset of the deliberately evoked slips (cf. Johansson and Westling, 1984a). This normal-to-tangential force ratio, termed the slip ratio, represented the inverse of the coefficient of static friction. It was on average $0.90 \pm 0.10$ (mean $\pm \mathrm{SD}$; data from all subjects and surface angles pooled) and was not influenced by the surface angle. However, it varied moderately among the subjects, being $0.74 \pm$ 0.07 and $1.0 \pm 0.09$ for the two extremes, respectively.

To assess the possible influence by digital anesthesia on friction, in one subject we measured the friction with and without anesthesia in a separate series of trials, presented according to the blocked design. The slip ratios were similar during normal $(0.81 \pm 0.11 ; n=40)$ and anesthetized conditions $(0.83 \pm 0.12 ; n=40)$, indicating that anesthesia did not substantially influence the friction with our sandpaper surface. Moreover, during the series with unpredictable variation in surface angle, we analyzed accidental slips associated with dropping of the object. Such slips happened exclusively in trials with a $30^{\circ}$ surface angle and mostly in trials with no vision. With normal digital sensibility, accidental dropping occurred only in about $3 \%$ of these trials but was more common during digital anesthesia (25\%). Again the estimated slip ratios during normal sensibility ( $0.95 \pm 0.1 ; n=13$; data pooled across subjects) were similar to those with anesthesia $(0.97 \pm 0.11 ; n=54)$. With other materials it is known that the friction may be lower during anesthesia, probably because of impaired sudomotor activity (Johansson and Westling, 1984a,b; Smith and Scott, 1996).

The safety margin against frictional slips was estimated during the static phase of each lift of the test series in which the surface angle was kept constant in blocks of consecutive trials. The safety margin was computed as the difference between the static normal force used and the minimum normal force required to prevent slip. A measure of this minimum force, termed the slip force, was obtained by multiplying the static tangential force and the slip ratio.

The preload phase (Fig. 1C, a) was defined for each trial from the moment that the first digit (thumb or index finger) contacted the object until the vertical force had reached $10 \%$ of the static vertical force. The moment of contact was determined for each digit and trial as the point in time when the rate of the horizontal force reached $2 \mathrm{~N} / \mathrm{sec}$, i.e., the minimum rate that could be detected reliably in our single trial records. The load phase (Fig. 1C, b) was defined as the period from the end of the preload phase until the vertical force had increased to $90 \%$ of the static vertical force. To characterize the force development during the initial dynamic phase of the lifts further, horizontal forces were measured when the vertical force was 10,50 , and $90 \%$ of the static vertical force, and the maximum horizontal force was measured as the peak force occurring during a one second interval starting at the end of the load phase (see Fig. 1C, left panel, VF10\%, VF50\%, VF90\%, Maxi- 
A

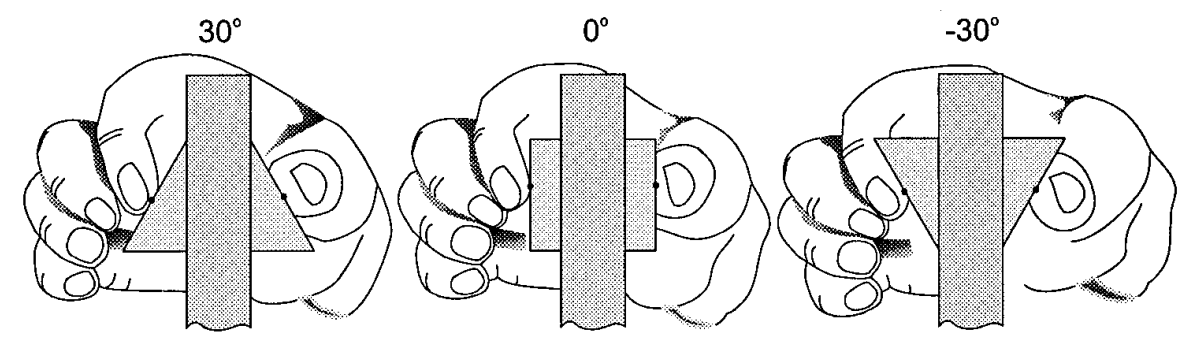

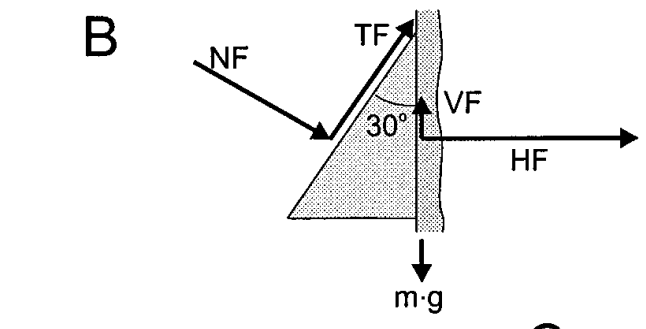

C

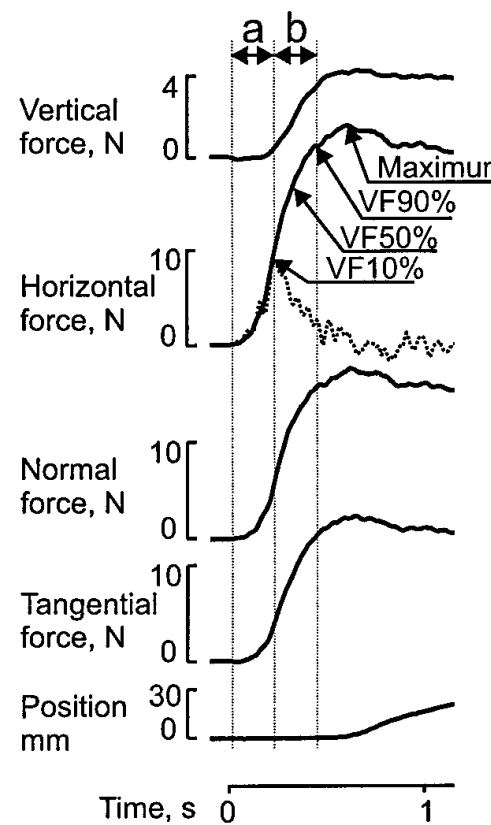

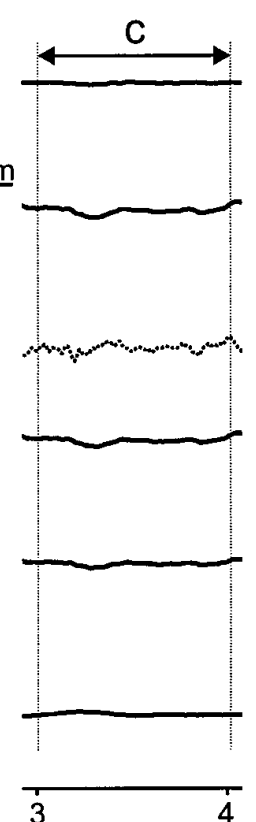
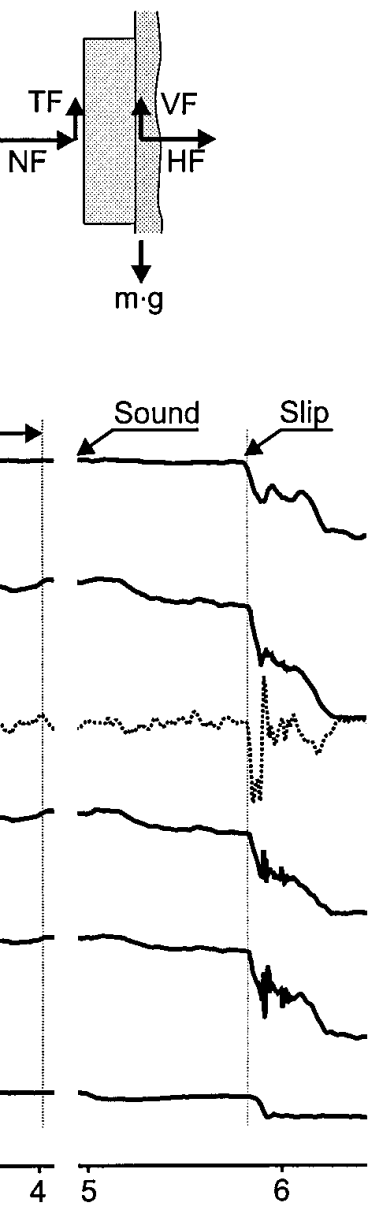
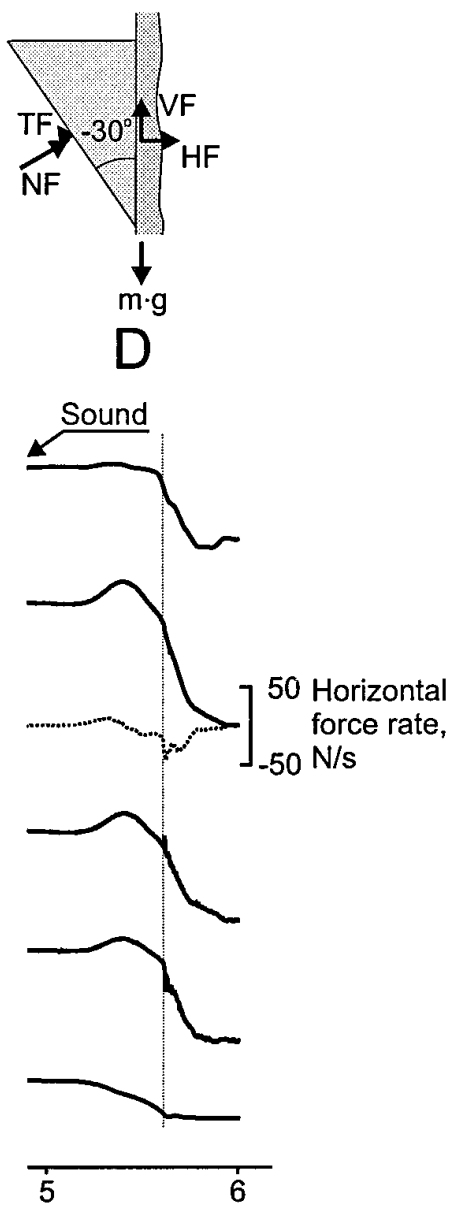

Figure 1. Instrumented test object and measurements taken for analyses. $A$, Orientation of the grip surfaces for three different surface angles: 30 , 0 , and $-30^{\circ}$. B, Vectorial representation of recorded $\mathrm{HF}$ and $\mathrm{VF}$ and computed $\mathrm{NF}$ and TF exemplified at surface angles of 30,0 , and $-30^{\circ}$. $C$, Fingertip forces and vertical movements of an object shown as a function of time for different phases of a lifting trial terminated by the subject slowly decreasing the grip force until slippage (arrow, slip) after a sound signal (arrow, sound). The intervals $a$ and $b$ indicate the preload phase and the load phase, respectively. Interval $c$ shows the period 3-4 sec after the object was initially touched while static phase measurements were taken. Arrows illustrate different points of measurements of horizontal force during the load phase, i.e., horizontal forces at 10, 50, and 90\%, of the static VF and maximum HF. D, End of a trial in which the subjects replaced the object on the support table in an ordinary manner after a sound signal (arrow, sound). The vertical line indicates contact with the support table.

mum). The static forces were calculated as the mean forces during the time interval 3-4 sec after the beginning of the lift, i.e., when the object was held still in air (Fig. 1C, $c$ ).

Statistical methods. For data gathered in the series in which the surface angle was kept constant in blocks of trials, repeated measures ANOVAs were used to evaluate the influence of angle $(-40,-30,-20,-10,0,10$, 20 , and $30^{\circ}$ ) on the following measures: (1) duration of the preload phase; (2) duration of the load phase; (3) the horizontal forces at load forces corresponding to 10,50 , and $90 \%$ of the static vertical force; (4) maximum horizontal force; (5) static horizontal force; (6) slip ratio; and (7) safety margin. For data gathered in lifting series with unpredictable changes in surface angles and with normal digital sensibility, repeated measures ANOVAs were used to evaluate the influence of vision (sighted or blind), angle $\left(-30,0\right.$, or $\left.30^{\circ}\right)$, and angle in the previous trial $(-30,0$, or $30^{\circ}$ ) on variables $1-5$ as indicated above. Finally, a separate set of ANOVAs was applied to data from the four subjects that participated in experiments with digital anesthesia. The influence of digital sensibility (normal or impaired), vision (sighted or blind), angle $\left(-30,0\right.$, or $\left.30^{\circ}\right)$ and angle in the previous trial $\left(-30,0\right.$, or $\left.30^{\circ}\right)$ were analyzed on the same five variables. A repeated measures design could not be used in this case because of the small number of subjects. For each ANOVA the level of probability chosen as statistically significant was $p<0.05$. All possible effects were not examined. Rather, the analyses focused on planned comparisons and specific effects as described in Results. Unless otherwise indicated, population estimates are presented in the form of mean \pm SD values and are based on data pooled across subjects. 

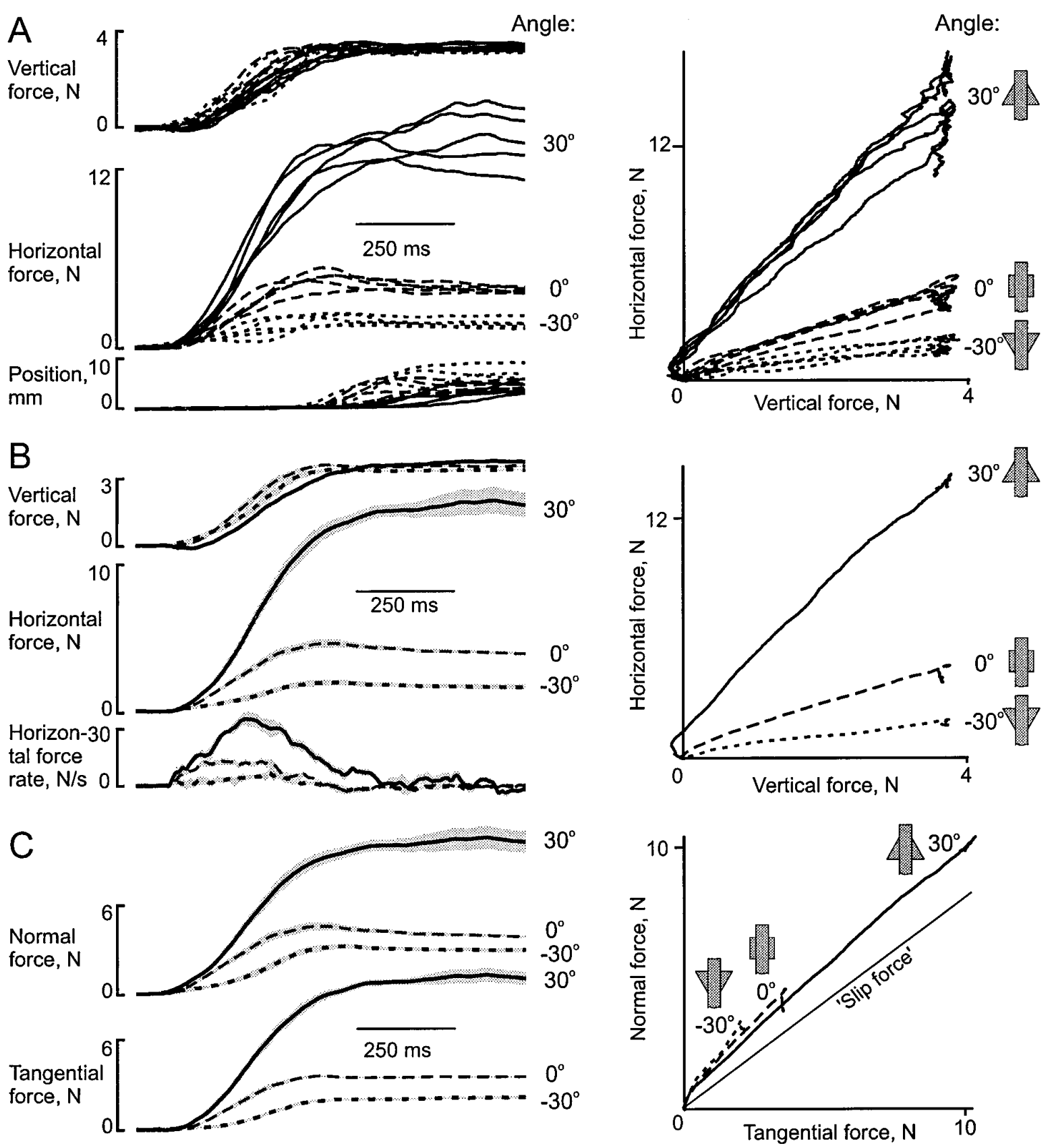

Figure 2. Force coordination during the initial part of lifts by a single subject with surface angles of $30^{\circ}$ (solid lines), $0^{\circ}$ (dashed lines), and $-30^{\circ}$ (dotted lines). Data are from lift series in which the surface angle was kept constant in blocks of five consecutive trials. A, Left panel, vertical and horizontal forces and vertical position as a function of time for all five consecutive trials (superimposed) with each surface angle. Right panel, coordination between these forces by displaying the horizontal force against the vertical force. $B$, Averaged vertical and horizontal forces and horizontal force rate for the same trials as in $A$. C, Averaged normal force and tangential forces for the same trials. Right panel, coordination between normal force and tangential forces by displaying the normal force against the tangential force. The solid line gives the minimum estimated normal force (Slip force) as a function of the tangential force; the vertical distance between this line and the curves represents the normal force safety margin against frictional slips. $B$, $C$, The shaded zones of the curves give \pm 1 SEM. $A-C$, All trials were synchronized in time on touch, i.e., when the horizontal force rate exceeded $2 \mathrm{~N} / \mathrm{s}$. In addition to the surface angle given in degrees, the object shape is illustrated by the shaded inset figures (compare Fig. $1 \mathrm{~A}$ ).

\section{RESULTS}

\section{Object shape controls fingertip forces}

We initially studied the fingertip forces used in a lift series while the shape of the object was kept constant in blocks of trials and the subjects saw the object and had normal digital sensibility. All subjects used progressively higher horizontal forces when the surface angle was increased, i.e., when the object became more tapered upward (Figs. $2 A, B, 3 A$ ). This effect of object shape was present throughout the trial, because the surface angle principally influenced the rate of horizontal force change (Fig. $2 A, B$ ). Thus, the horizontal forces at 10,50 , and $90 \%$ of the static vertical force before lift-off and the maximum and static horizontal forces all varied with the surface angle $(p<0.0001$ in each instance; Fig. $3 B)$. The generation of vertical force and the vertical movement 

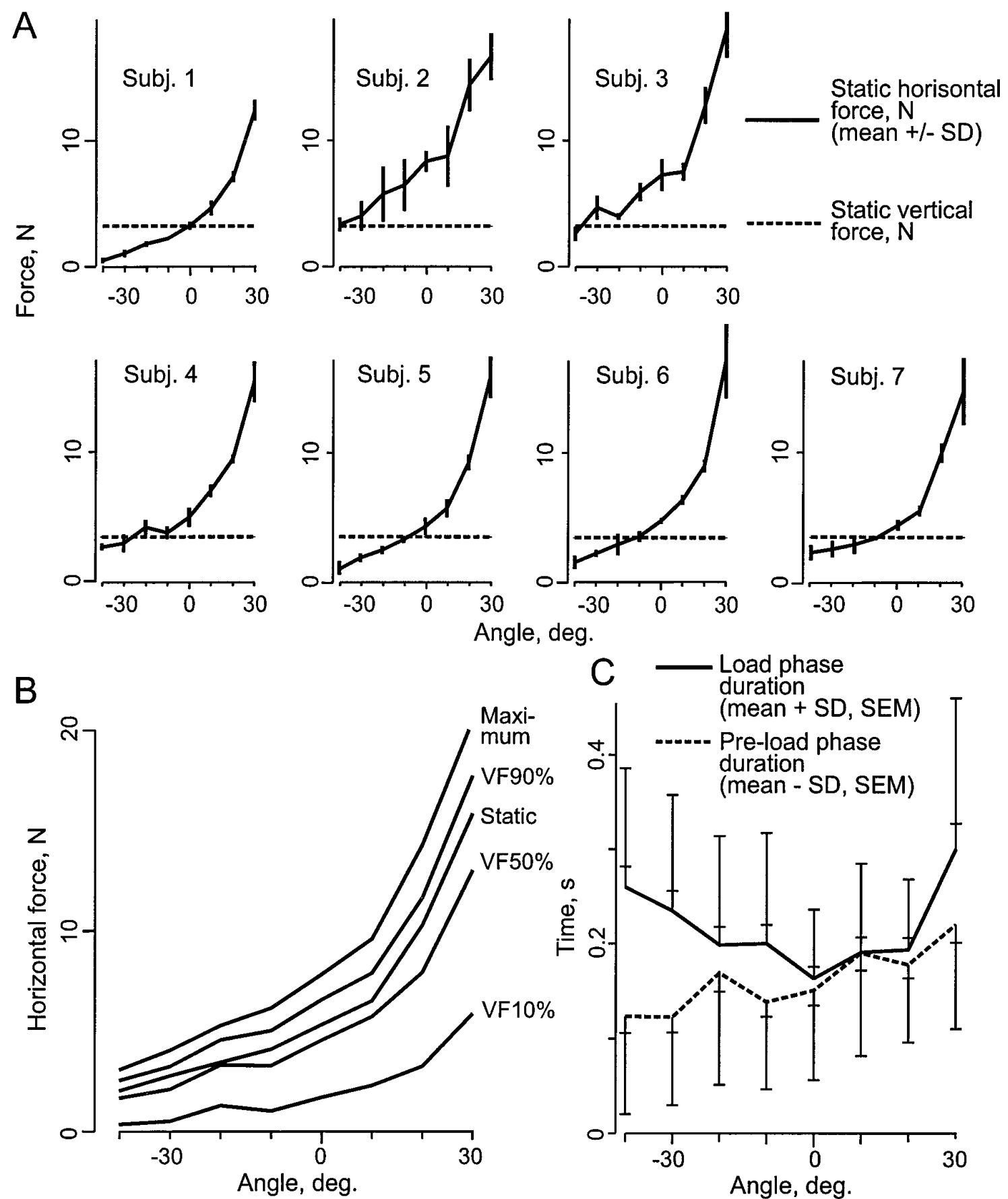

Figure 3. Horizontal forces and duration of preload and load phases during lift series in which each surface angle was presented in blocks of five consecutive trials. $A$, Static horizontal force (solid line) and static vertical force (dotted line) plotted against surface angle. Mean forces \pm SD are illustrated for each subject (Subj. 1-7). B, Horizontal force at vertical forces corresponding to 10, 50, and $90 \%$ of the static vertical force, static horizontal force (Static), and maximum horizontal force plotted against the angle. Curves represent average values for all seven subjects. $C$, Mean duration of preload and load phases plotted against surface angle; 1 SD and 1 SEM are unilaterally indicated for data averaged across all seven subjects.

were less influenced by the surface angle; the static vertical force was not influenced at all, because the weight of the object was constant. Consequently, the balance between the vertical force and horizontal forces was influenced by the surface angle; i.e., the higher the angle, the stronger the horizontal force at any given vertical force (Fig. $2 A, B$, right panels).

The modest influence of surface angle on the generation of vertical force partly concerned its rate of increase during the load phase, i.e., during the period of isometric force development when the vertical force increased from 10 to $90 \%$ of the static vertical force. There was a gradual prolongation of the duration of the load phase the more the surface angle deviated from $0^{\circ}(p<$ 0.005 ; Fig. $3 C$ ), indicating a lower rate of vertical force increase as the object tapered more upward or downward. Also, the duration of the preload phase was influenced by the surface angle $(p<$ 0.02 ). It increased with increasing surface angle (Fig. 3C). This effect, however, could largely be explained by a mechanical coupling between the horizontal and vertical forces that depended on 

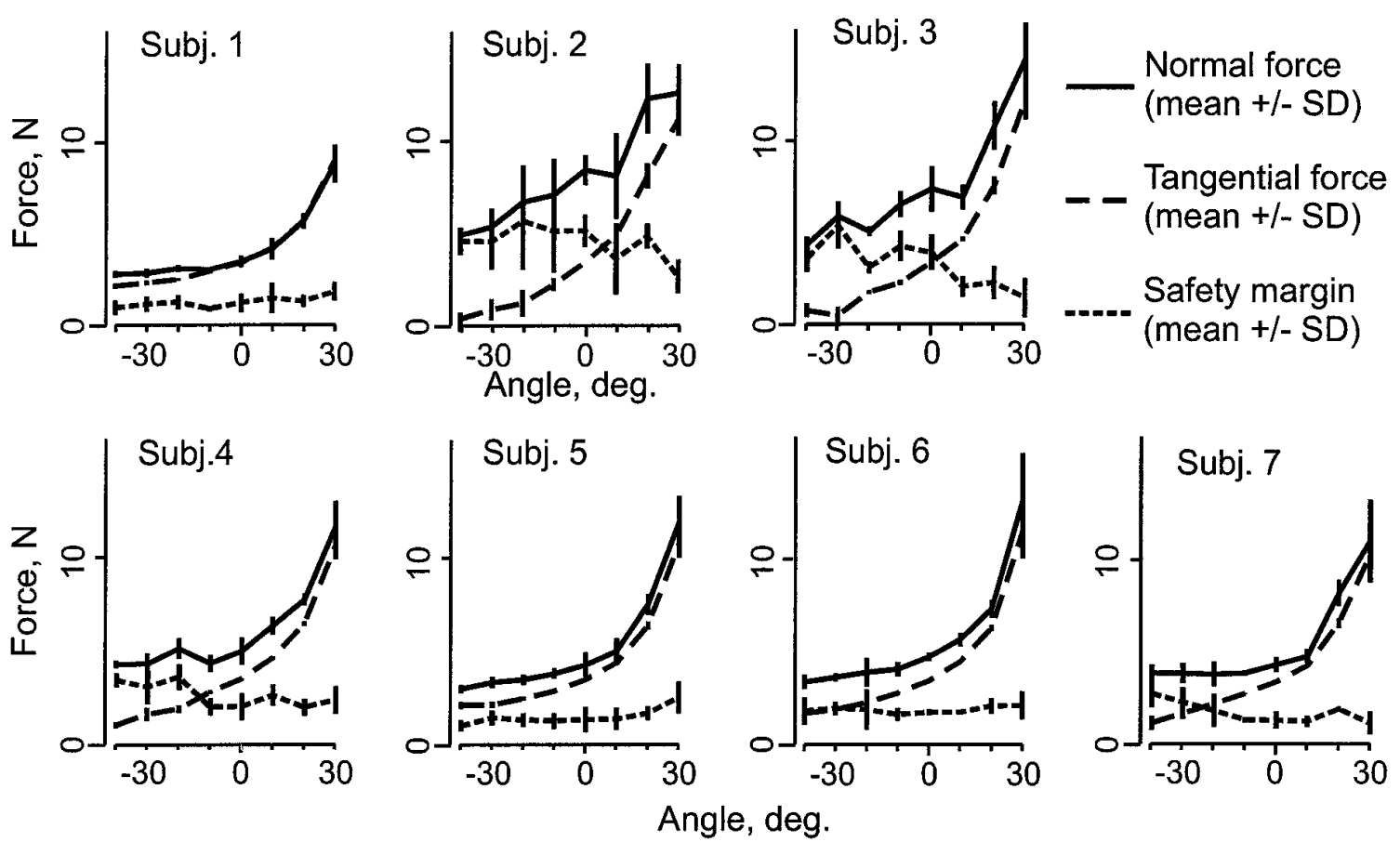

Figure 4. Static normal force (dashed lines), tangential force (solid lines), and normal force safety margin against frictional slip (dotted lines) as a function of surface angle. Mean values \pm SD are illustrated for all individual subjects (Subj. 1-7) who performed lift series in which each surface angle was presented in blocks of five consecutive trials.

the tapering of the grip surfaces. With downward-tapered grip surfaces (negative surface angles), the increase in horizontal force after the subject contacted the object contributed to a vertical force caused by asymmetric deformation of the fingertips. Because this vertical force was directed upward, it contributed to the vertical lift force and thus an early onset of the recorded increase in vertical force. Similarly, with upward-tapered grip surfaces (positive surface angles), the initial increase in horizontal force contributed to a downward-directed vertical force that counteracted the increase in the vertical lift force applied by the subject, which therefore seemed delayed. In fact, with positive angles the vertical force often took negative values during the initial period of the horizontal force increase (Fig. 2B; also see Figs. 5A, 9A).

The magnitudes of forces normal and tangential to the grip surfaces were markedly influenced by the surface angle throughout the lifting trials (Fig. 2C). These forces progressively increased as a function of the surface angle as a consequence of the manner in which the subjects changed the balance between the horizontal and vertical forces with the geometry of the object (Figs. $2 C, 4$ ). Importantly, throughout the lift trials subjects maintained a nearly constant balance between the normal and tangential forces, regardless of surface angles (Fig. $2 C$, right panel). Consequently, at any given tangential force the safety margin against frictional slips was similar for the various surface angles in terms of normal forces used in excess of the minimum normal force required to prevent slippage (compare the vertical distance between the "slip force" line and the curves in Fig. 2C). Likewise, during the static hold phase of the lifts the normal force safety margin was similar over the entire angular range (Fig. 4). It was $2.7 \pm 1.7$ and $2.0 \pm$ $0.9 \mathrm{~N}$ at -30 and $30^{\circ}$, respectively. However, if expressed as the fraction of the static normal force used, the safety margin decreased considerably with surface angle; it was $61 \pm 21$ and $17 \pm$ $7 \%$ at $-30^{\circ}$ and $30^{\circ}$, respectively. As observed in earlier studies of lifting tasks, the safety margin against slippage varied between subjects (Fig. 4) (Westling and Johansson, 1984).

\section{Adaptation of force coordination to changes in object shape}

Using data obtained in test series with unpredictable variation of surface angle (between $-30,0$, and $30^{\circ}$ ) we examined the adaptation of the fingertip forces to changes in object shape, with emphasis on sensory cues used by the subjects. Vision may have contributed as well as somatosensory inputs from the digits after the object was contacted. To assess the relative importance of these and other possible sensory sources, we compared the subjects' performance with and without vision and during normal and impaired digital sensibility. Furthermore, to evaluate the possible role of anticipatory parameter control based on previous experience with the object (see the introductory remarks), we specifically analyzed influences of the surface angle in previous trials.

\section{Lifts with vision and normal digital sensibility}

In series of trials in which the subject saw the test object (and the hand), the surface angle influenced the development of horizontal force even at the onset of force application (compare Fig. 2). This was true not only for trials in which the surface angle was the same as in the previous trial but also for trials performed after a change in surface angle. Thus, in this condition object shape controlled the force output from the beginning of the lifting trial.

Figure 5, $A$ and $B$, compares the time course of force development in the dynamic phase of trials carried out subsequent to a change in surface angle, with the force development in trials with the same surface angle not preceded by such a change. The adjustment to a smaller angle is illustrated in Figure $5 \mathrm{~A}$. The solid curves represent trials with a surface angle of $-30^{\circ}$ preceded by trials with an angle of $30^{\circ}$ (Fig. $5 A, 30^{\circ} \rightarrow-30^{\circ}$ ); i.e., trials carried out with the object tapered downward after a change from an 
With vision

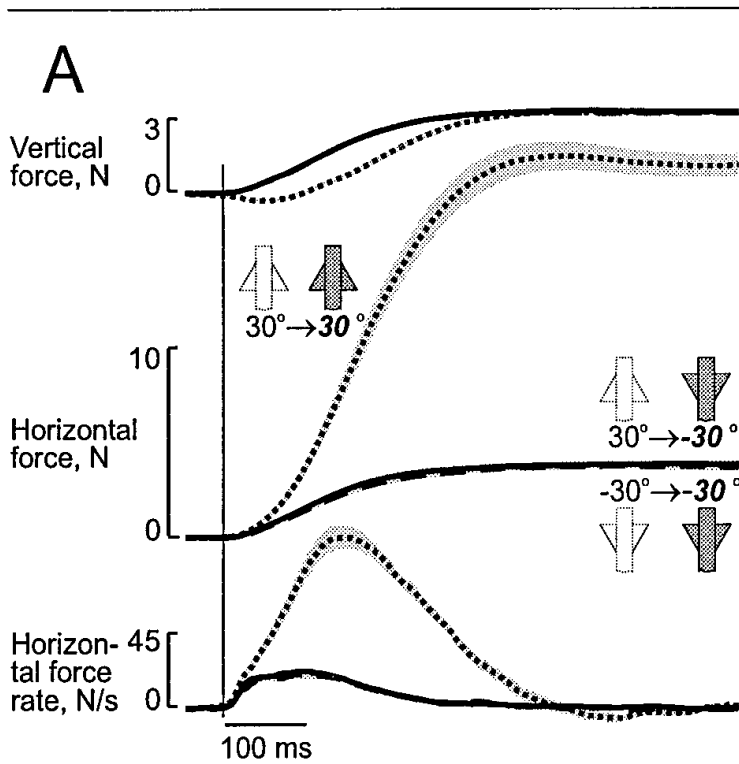

B

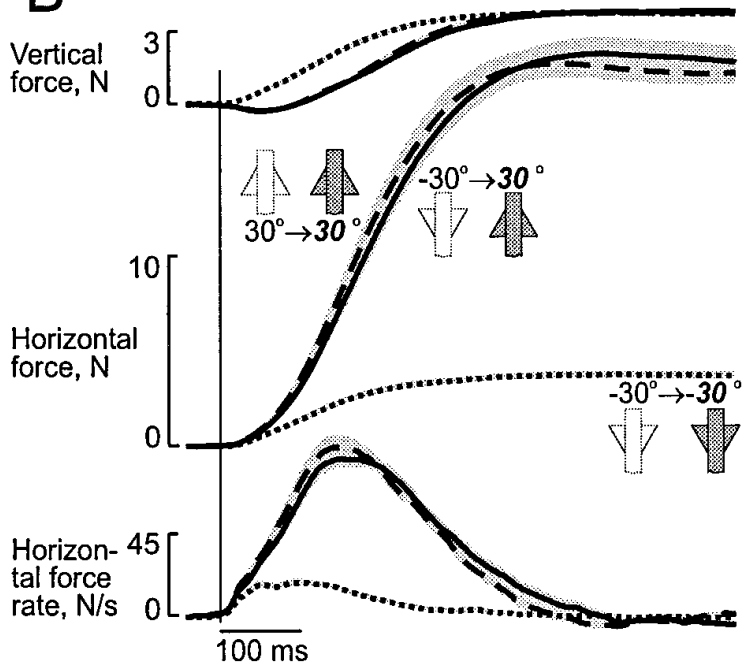

Without vision
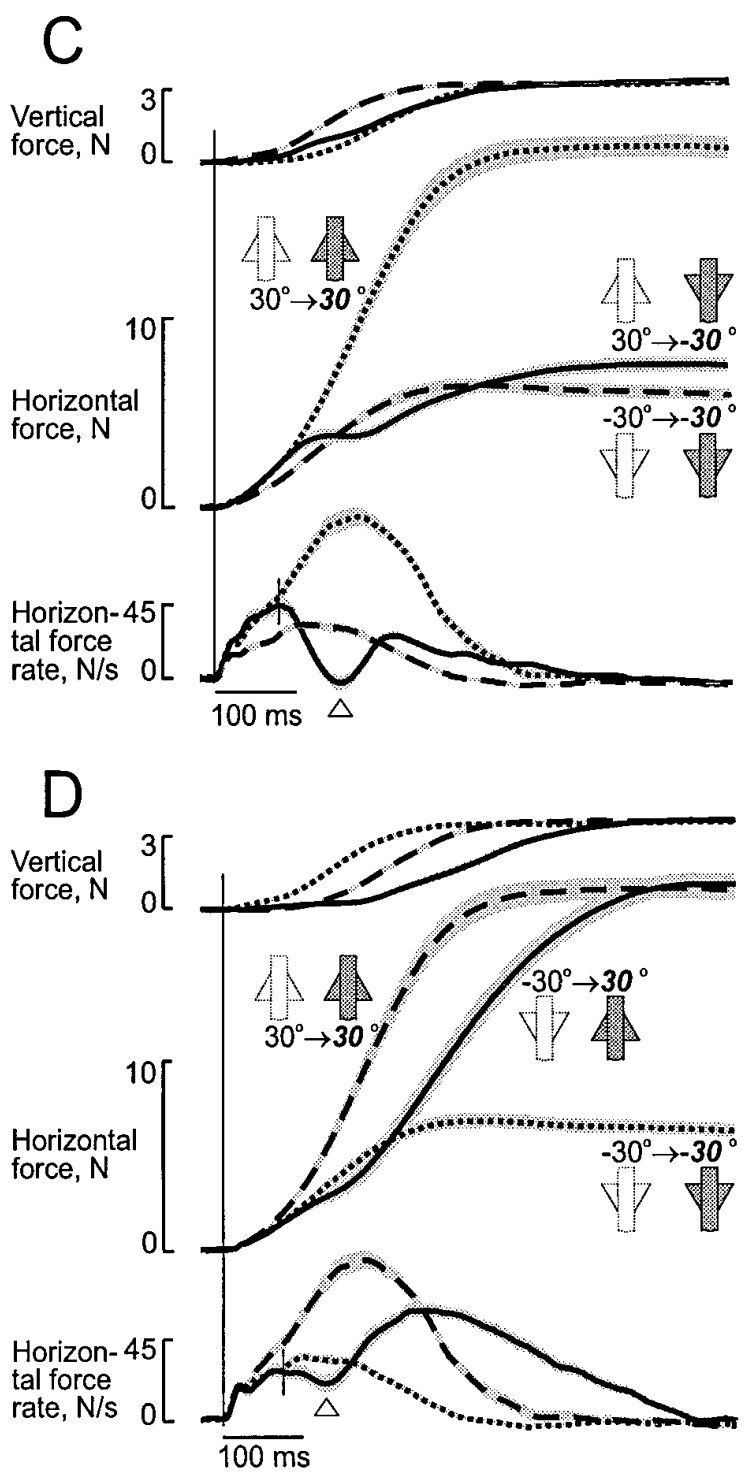

Figure 5. Adjustments to changes in surface angle during lift series in which object shape was unpredictably varied between trials. Data are averaged from eight subjects with normal digital sensibility and who showed similar load phase durations; single trials were synchronized in time when the horizontal force rate exceeded $2 \mathrm{~N} / \mathrm{s}$. Vertical and horizontal forces and horizontal force rate as a function of time for trials with vision $(A, B)$ and without vision $(C, D)$ are shown. The shaded zones of the curves give \pm 1 SEM, and the vertical line indicates the start of horizontal force increase. In addition to surface angle given in degree, the object shapes in current and previous trials are illustrated by the shaded and open inset figures, respectively (compare Fig. $1 A$ ). $A, C$, Adjustment to a smaller angle is illustrated by trials with $-30^{\circ}$ preceded by trials with $30^{\circ}\left(30^{\circ} \rightarrow-30^{\circ}\right.$, solid line $)$. Trials with $-30^{\circ}\left(-30^{\circ} \rightarrow-30^{\circ}\right.$, dashed line) and $30^{\circ}\left(30^{\circ} \rightarrow 30^{\circ}\right.$, dotted line $)$ not preceded by a change in surface angle are shown for comparison. $B, D$, adjustment to a larger angle is illustrated by trials with $30^{\circ}$ preceded by trials with $-30^{\circ}\left(-30^{\circ} \rightarrow 30^{\circ}\right.$, solid line $)$. Again, trials with $-30^{\circ}\left(-30^{\circ} \rightarrow-30^{\circ}\right.$, dotted line $)$ and $30^{\circ}\left(30^{\circ} \rightarrow 30^{\circ}\right.$, dashed line) not preceded by a change in surface angle are shown for comparison. $C, D$, Short vertical lines indicate points in time at which the new surface angle was expressed in the motor output. Arrowheads indicate the reduced rate force occurring before the horizontal force again increased toward a level adequate for the current surface angle.

upward-tapered shape. Importantly, the force development in these $-30^{\circ}$ trials was identical to that in trials with $-30^{\circ}$ preceded by trials with $-30^{\circ}$ (Fig. $5 A,-30^{\circ} \rightarrow-30^{\circ}$, dashed curves) and clearly different from that during the preceding trials with an angle of $30^{\circ}$, which in turn had been preceded by trials with an angle of $30^{\circ}$ (compare Fig. $5 \mathrm{~A}, 30^{\circ} \rightarrow 30^{\circ}$, dotted curves). Thus, the force output was adapted to the current "new" angle at the onset of the horizontal force increase.

The adjustment to a more positive angle is illustrated in Figure $5 B$ for trials with a surface angle of $30^{\circ}$ that were preceded by trials with an angle of $-30^{\circ}\left(-30^{\circ} \rightarrow 30^{\circ}\right.$, solid curves $)$. Again the force output was adapted to the new angle at the onset of the horizontal force increase (Fig. 5B, compare dashed and solid curves) with little influences from the preceding $-30^{\circ}$ trials (Fig. $5 B$, compare dotted and solid curves).

These findings strongly suggest that subjects used visual information about object shape in a feed-forward manner to adapt the force output to the shape of the object. Furthermore, visual memory related to the angle of the previous trial did not significantly influence the balance between the horizontal and vertical 


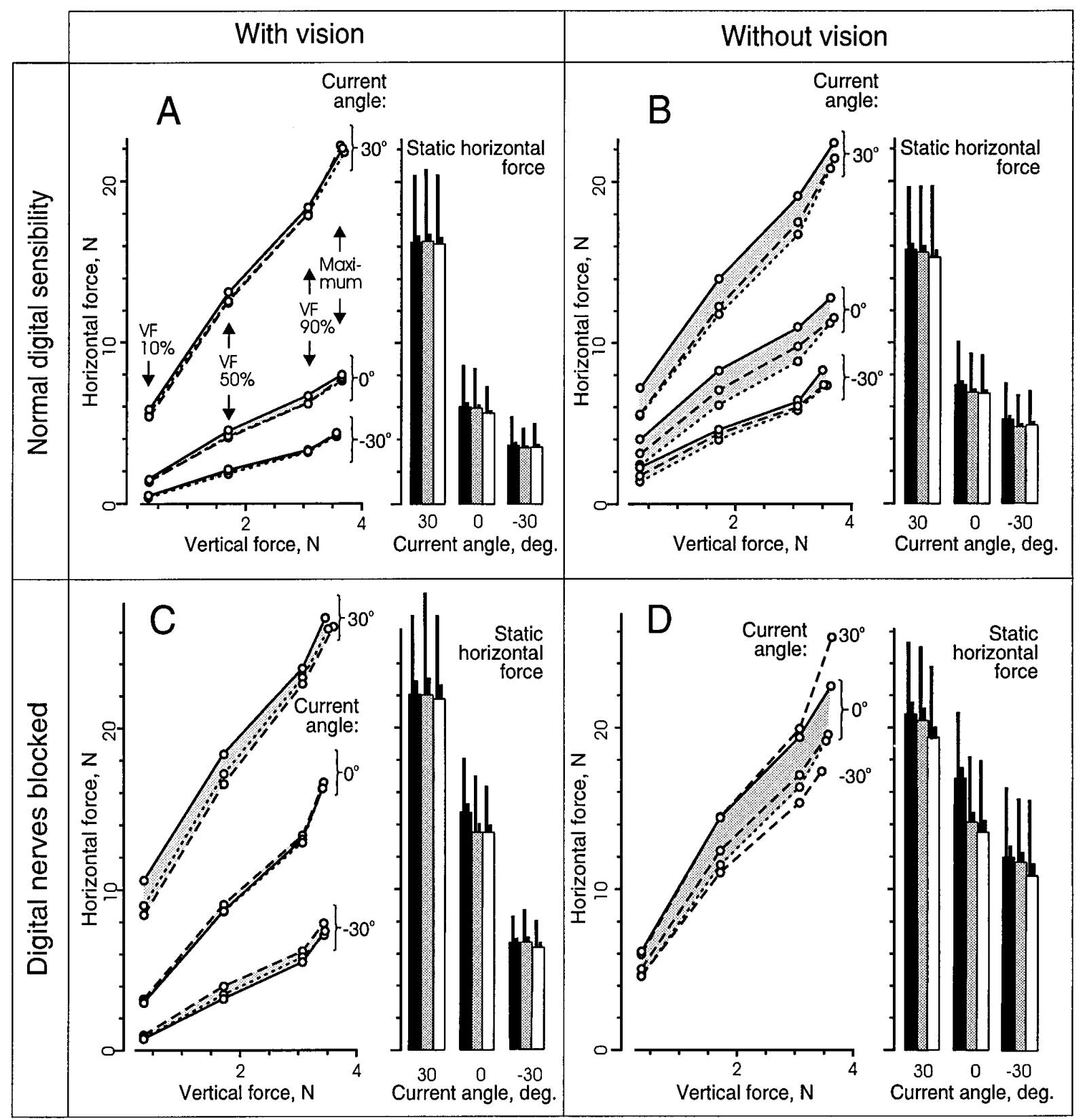

Previous angle $=30^{\circ}$

Previous angle $=0^{\circ}$

$\square$ Previous angle $=-30^{\circ}$

Figure 6. Coordination of horizontal and vertical forces and effects of surface angle in the previous trial for trials with vision $(A, C)$ and without vision $(B, D)$. Subjects had normal digital sensibility in $A$ and $B$ and impaired digital sensibility in $C$ and $D$. $A-D$, The left graph in each panel indicates mean horizontal forces at vertical forces corresponding to 10,50 , and $90 \%$ of static vertical force and maximal horizontal force as a function of vertical force. Shaded areas indicate data obtained with a given surface angle $\left(0,30\right.$, or $\left.-30^{\circ}\right)$; solid, dashed, and dotted lines refer to the surface angle of the preceding trial, i.e., 0,30 , and $-30^{\circ}$, respectively. (For clarity, effects by the previous trials are shown only for trials with a $0^{\circ}$ angle in $D$; i.e., there was a great overlap between data obtained during the dynamic phases of trials by different surface angles in this condition.) The histograms in each panel represent mean static horizontal forces at each surface angle and the influences of the preceding trial: $0^{\circ}$ (shaded columns), $30^{\circ}$ ( filled columns), and - $30^{\circ}$ (open columns). The pair of vertical bars at the top of each column gives +1 SD and +1 SEM, respectively. Data are pooled from all subjects who performed series with unpredictable variation in surface angle between trials.

forces during any part of the lifts (Fig. 6A); only the prevailing angle did so.

It is possible that subjects used vision to identify which of the three object shapes was presented (corresponding to $-30,0$, and $30^{\circ}$ surface angles) and then retrieved information about the required finger forces from memory of previous lifts with these specific shapes (cf. scaling of the force output to the weight of common objects in Gordon et al., 1993). Alternatively, subjects may have used vision in a "computational" sense, relying on implicit general knowledge about relationships of the shapes of objects and required force coordination. That is, visual cues about the surface angle may have been used to compute the required balance between horizontal and vertical forces directly, without relying on previous experience from the particular object shapes 
that were presented. The present experiments indicate that subjects indeed used vision in the computational sense; with vision all subjects adequately adapted the force output to the shape at the first trial in which a new object shape was encountered. This was most convincingly shown in the lift series in which the surface angle was kept constant in blocks of consecutive trials (see Materials and Methods). There were no statistically significant differences between the forces used in the first and last trials of each series. This was tested by a repeated measures $8 \times 2$ multivariate ANOVA in which surface angle $(n=8)$ and first versus last trial $(n=2)$ were represented as fixed effects on the horizontal forces measured at 10,50 , and $90 \%$ of static vertical force, maximum horizontal force, and static horizontal force $(p>0.27$ for first vs last trial). Thus, approximately the same coordination between the horizontal force and the vertical force was maintained throughout all trials of each block, indicating that there was no learning involved during a block.

\section{Lifts without vision but with digital sensibility}

Although visual information seemed to be an important sensory source for adapting the force output to object shape, all subjects efficiently adapted the force output to object shape even when they were blindfolded. However, throughout the lifting trials with -30 and $0^{\circ}$ surface angles, subjects used higher horizontal forces than with vision $(p<0.03$ for each measure of horizontal force at 10,50 , and $90 \%$ of static vertical force and for maximum horizontal force and static horizontal force; Fig. 6, compare $A$ and $B$ ). Consequently, provided that the friction was similar without vision, safety margins were larger with these surface angles, which demanded smaller fingertip forces than with the $30^{\circ}$ angle.

Although there was no significant main effect of vision (sighted or blind) on the load phase duration, it seem slightly prolonged in the blindfolded condition (Fig. 7A). Furthermore, only the blindfolded condition trials performed after a change in surface angle showed a prolonged duration of the load phase compared with trials preceded by lifts with the same surface angle $(p<0.007$; Fig. 7B).

Figure 5, $C$ and $D$, shows the adjustment to a new surface angle during blindness. The adjustment to a smaller angle is illustrated in Figure $5 \mathrm{C}$ for trials with $-30^{\circ}$ preceded by trials with $30^{\circ}$ $\left(30^{\circ} \rightarrow-30^{\circ}\right.$, solid curves $)$. During the first $70-90 \mathrm{msec}$ after the initial contact with the object, the development of horizontal force was similar to that in the preceding $30^{\circ}$ trials, which in turn were preceded by trials with $30^{\circ}$ (Fig. 5C, compare trails labeled $30^{\circ} \rightarrow 30^{\circ}$, dotted curves ). That is, in contrast to corresponding trials carried out with vision, the $-30^{\circ}$ trial was initially executed as if there had been no change in surface angle (Fig. 5, compare $A$ and $C)$. An adjustment of the force coordination to the new angle began after $70-90 \mathrm{msec}$, when the rate of horizontal force increase slowed compared with that in the preceding $30^{\circ}$ trials (Fig. $5 C$, compare solid and dashed curves).

The adjustments to a more positive angle are illustrated in Figure $5 D$ for trials with a surface angle of $30^{\circ}$ that were preceded by trials with an angle of $-30^{\circ}\left(-30^{\circ} \rightarrow 30^{\circ}\right.$, solid curves $)$. Again, without vision the initial $70-90 \mathrm{~ms}$ period of horizontal force increase was similar to that with $-30^{\circ}$ trials preceded by $-30^{\circ}$ (Fig. $5 D,-30^{\circ} \rightarrow-30^{\circ}$, dotted curves). After this period the development of the horizontal force output diverged compared with that in the previous $-30^{\circ}$ trial, reflecting the onset of an adjustment to the new surface angle.

As indicated by arrowheads in Figure 5, $C$ and $D$, during adjustments to smaller and larger surface angles the initial force
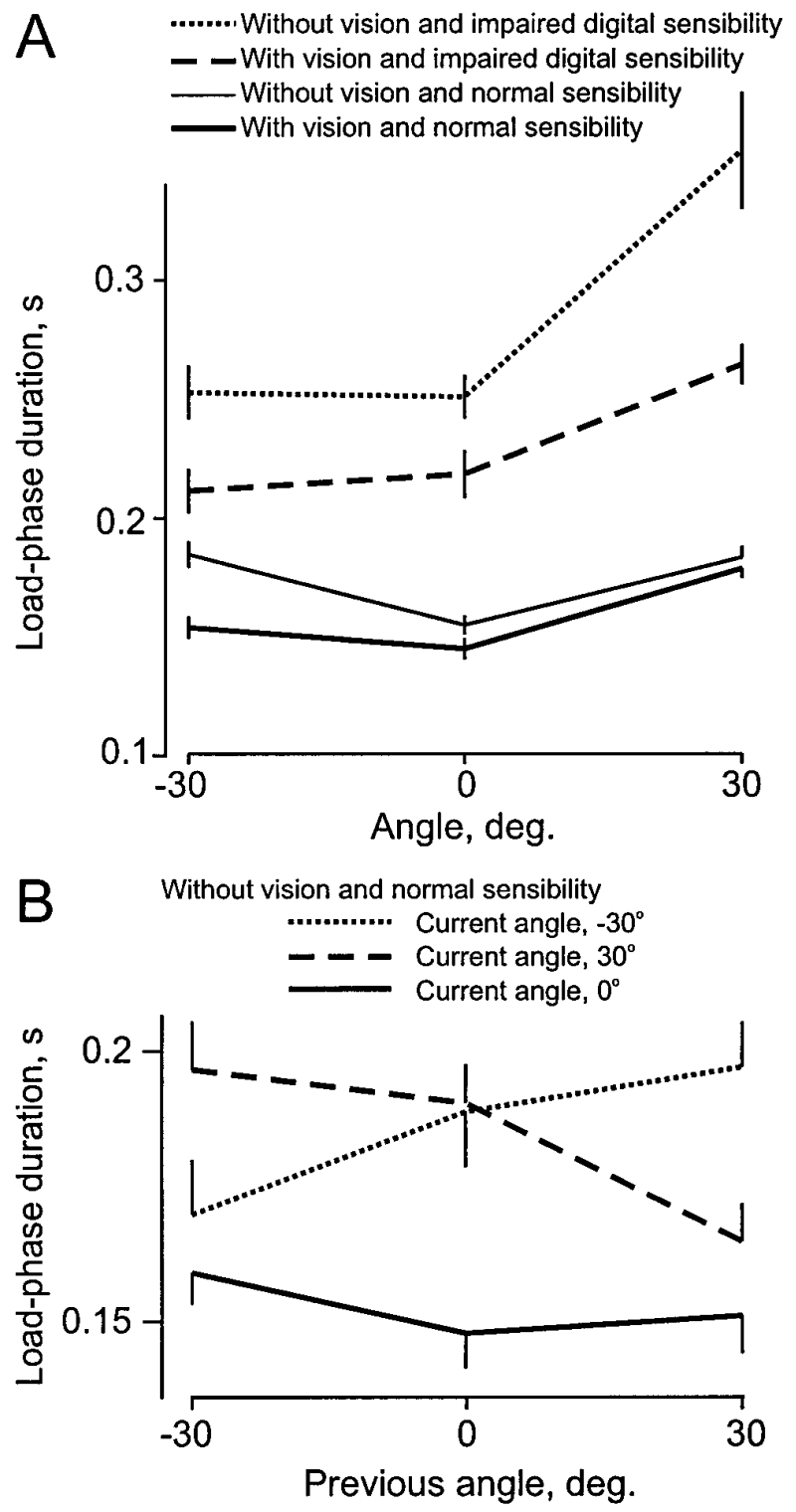

Figure 7. Influences on load phase duration by surface angle and various experimental conditions. $A$, Load phase duration as a function of surface angle in trials with and without vision and with and without digital nerve blocks. Pooled data are from all trials by the subjects who participated in lift series with unpredictable variation in surface angle between trials. $B$, Influences of surface angle in a previous trial on load phase duration. Pooled data are from all trials without vision but with normal digital sensibility by the subjects participating in lift series with unpredictable variation in surface angle. $A, B$, Vertical bars represent SEM, and curves give mean values.

increase seemed to be markedly reduced before a new command was executed that generated forces adequate for the current surface angle. The adjustment of force coordination also followed similar patterns with smaller changes in surface angle. This is illustrated in Figure 8 for transitions from 0 to $-30^{\circ}$ and 0 to $30^{\circ}$ angles based on data from single subjects. However, in these instances a termination of the initial increase in horizontal force, as indicated by arrowheads in Figure 5, $C$ and $D$, was less obvious, and with transitions from 0 to $30^{\circ}$ the initial signs of the adjustment to the new angle could seem a bit later.

Somatosensory information thus mediated force coordination adjustments to changes in object shape soon after the object was 

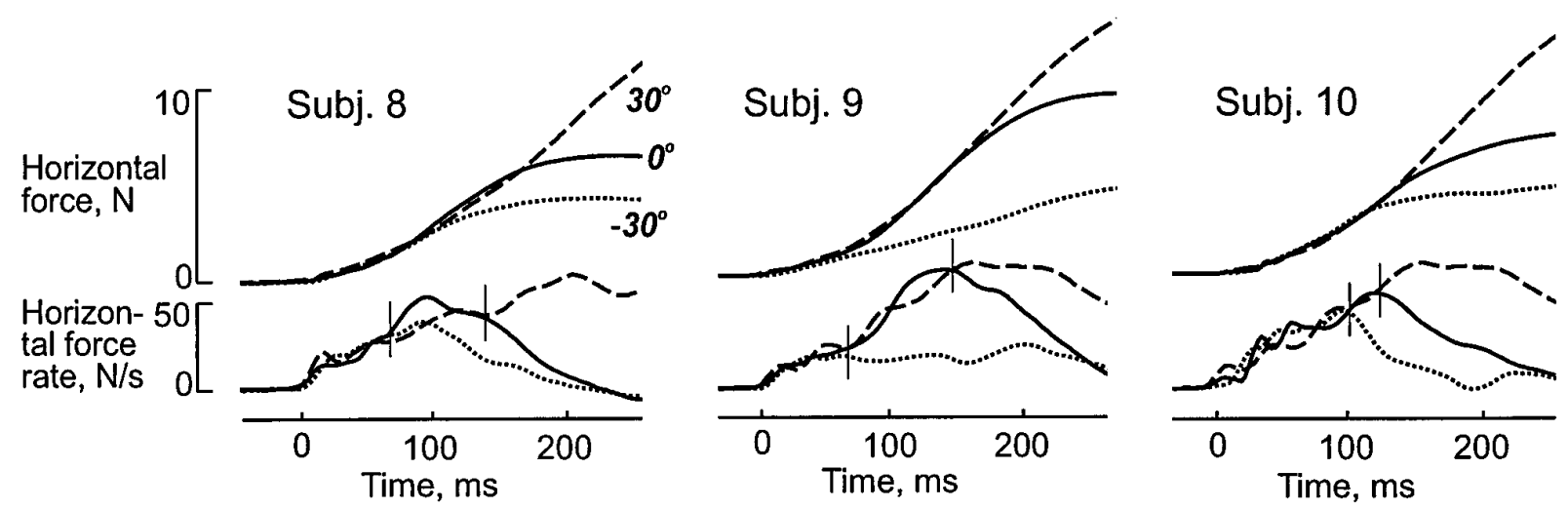

Figure 8. Adjustment to a change in surface angle, from 0 to $-30^{\circ}$ and from 0 to $30^{\circ}$ by blindfolded single subjects (Subj. 8-10), during normal digital sensibility. Horizontal force and its rate as a function of time are shown for trials carried out with $-30^{\circ}$ (dotted line), $0^{\circ}$ (solid line), or $30^{\circ}($ dashed line) angle. In all cases the surface angle in the previous trial was $0^{\circ}$. Thus, solid lines represent reference trials with a $0^{\circ}$ angle, which were preceded by trials with the same angle. Short vertical lines indicate points in time at which the new surface angle $\left(-30\right.$ or $\left.30^{\circ}\right)$ was expressed in the motor output, as judged from a comparison with the $0^{\circ}$ trials. Each curve represents average data from nine trials synchronized on start of horizontal force increase when the horizontal force rate exceeded $2 \mathrm{~N} / \mathrm{s}$.

touched in the absence of vision. However, these adjustment were not complete in the sense that memory related to object shape in the previous trial influenced the balance between the horizontal and vertical forces in the dynamic phase of the trials $(p<0.006$ for each measure of horizontal force at 10,50 , and $90 \%$ of static vertical force and for maximum horizontal force). The horizontal forces were stronger with a larger surface angle in the previous trial and weaker with a smaller surface angle (Fig. 6B). However, this effect by the previous trial was weak compared with the effect by the current surface angle and was smallest in trials with $-30^{\circ}$, which required small fingertip forces (Fig. 6B). We did not observe effects by the object shape in the previous trial in the static phase.

\section{Lifts with vision but without digital sensibility}

The behavior in sighted subjects whose digits were completely anesthetized further indicated that vision could control the force output in a feed-forward manner. These subjects all adapted the fingertip forces used to object shape (Fig. 9A). The surface angle influenced the force output at the onset of the force generation. As in experiments with vision and normal digital sensibility, the surface angle in the previous trial did not influence the force output. However, compared with normal digital sensibility the subjects used considerably stronger horizontal forces throughout the trials (Fig. 6, compare $A$ and $C)(p<0.0001$ for each measure of horizontal force at 10,50 , and $90 \%$ of static vertical force and for maximum and static horizontal force). Thus, the safety margins used against frictional slips would have been correspondingly larger, because the friction in the digit-object interface was similar to that during normal sensibility (see Materials and Methods). Furthermore, the load phase of the lifting trials was prolonged during anesthesia because of a slower force generation $(p<$ 0.0001) (Fig. 7A).

\section{Lifts without vision and without digital sensibility}

It is likely that the relevant somatosensory information originated from digital afferents, e.g., tactile afferents sensing the deformation of the digital skin as it molds to the geometry of the object. Indeed, the adaptation of the force coordination was severely impaired during a combination of blindfolding and digital anesthesia (Figs. 6D, 9B). The surface angle did not statistically influence the force coordination during the dynamic phase of the lift but did so during the static phase $(p<0.0001)$.

At $-30^{\circ}$ and $0^{\circ}$ surface angles the subjects used stronger horizontal forces than in any other experimental condition (Fig. 6). In contrast, frictional slips attributable to horizontal forces that were too low often occurred at the $30^{\circ}$ angle. These slips most frequently took place during the load phase; the object remained on the table in about $25 \%$ of the lifting trials with the $30^{\circ}$ angle. This problem, however, was overcome by the subject consciously attending to the firmness of the grip and by increasing the applied forces during subsequent lifting attempts until the object was lifted. This voluntary intervention with the force coordination evinced itself as an increased horizontal force rate during the load phase, resulting in a new force balance that tended to be maintained in subsequent trials. Thus, this course of action could explain the high horizontal forces for trials carried out with $0^{\circ}$ and $-30^{\circ}$ angles (Figs. $6 \mathrm{D}$ and $9 B$ ). Although subjects tended to keep up the horizontal forces after trials with slippage, the magnitude of this aftereffect decayed across the subsequent trials (cf. frictional changes during digital anesthesia in Westling and Johansson, 1984). The lower horizontal forces recorded in trials with $0^{\circ}$ and $-30^{\circ}$ angles than with a $30^{\circ}$ angle may partly be explained by this decay (Fig. 9B).

This aftereffect also indicated that the force coordination could be set via a memory trace during digital anesthesia. Accordingly, the horizontal forces were stronger with larger surface angles in the previous trial with anesthesia (see data from trials with a $0^{\circ}$ angle in Fig. 6D). But the effect of the surface angle in the previous trial was not limited to the dynamic part of the trials, as with blindfolded subjects with normal sensibility, but was present also during the static phase $(p<0.001$ for each measure of horizontal forces at 10,50 , and $90 \%$ of static vertical force and for maximum and static horizontal forces).

Finally, without vision and with digital nerve blocks, the load phase was further prolonged compared with that during digital anesthesia alone $(p<0.0001)$ (Fig. 7A). That is, there was a considerable slowing of the force generation compared with trials with normal sensibility but also a slowing compared with trials when vision was available during digital anesthesia. 

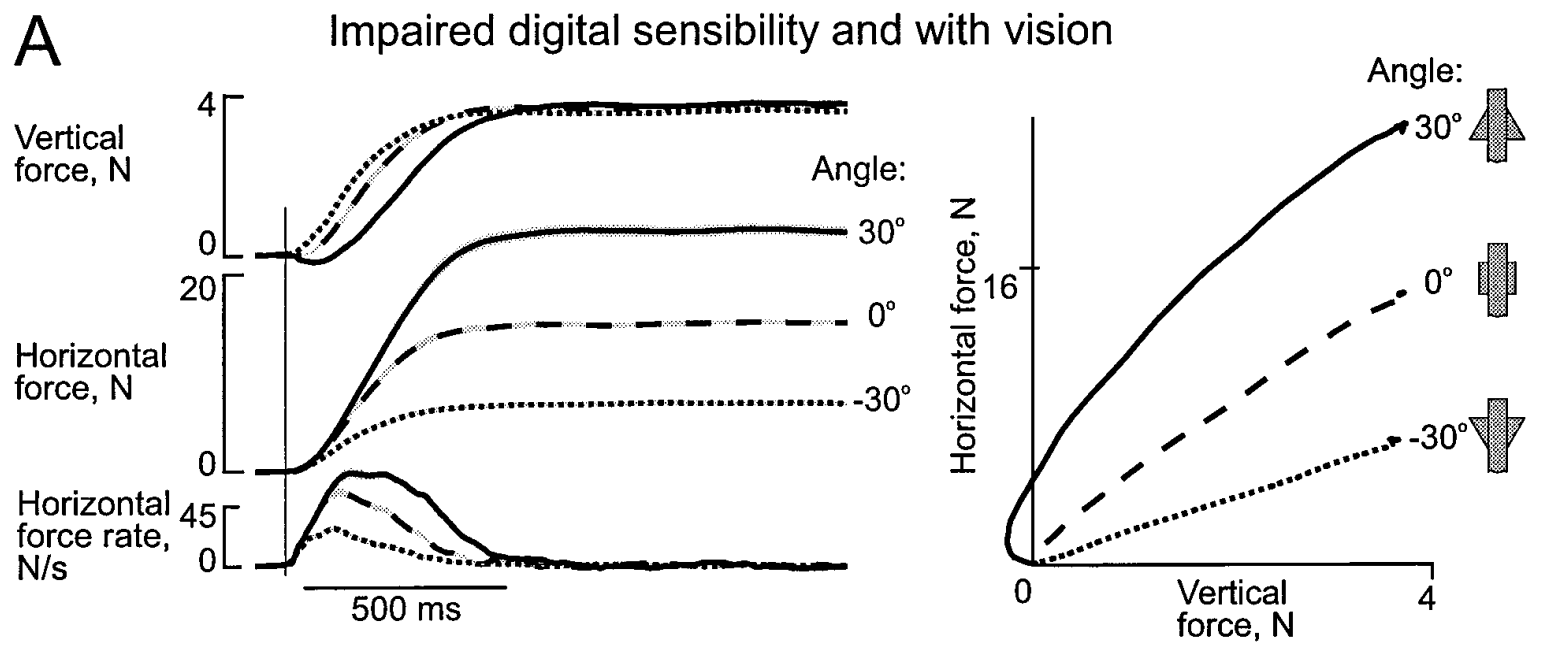

B

Impaired digital sensibility and without vision
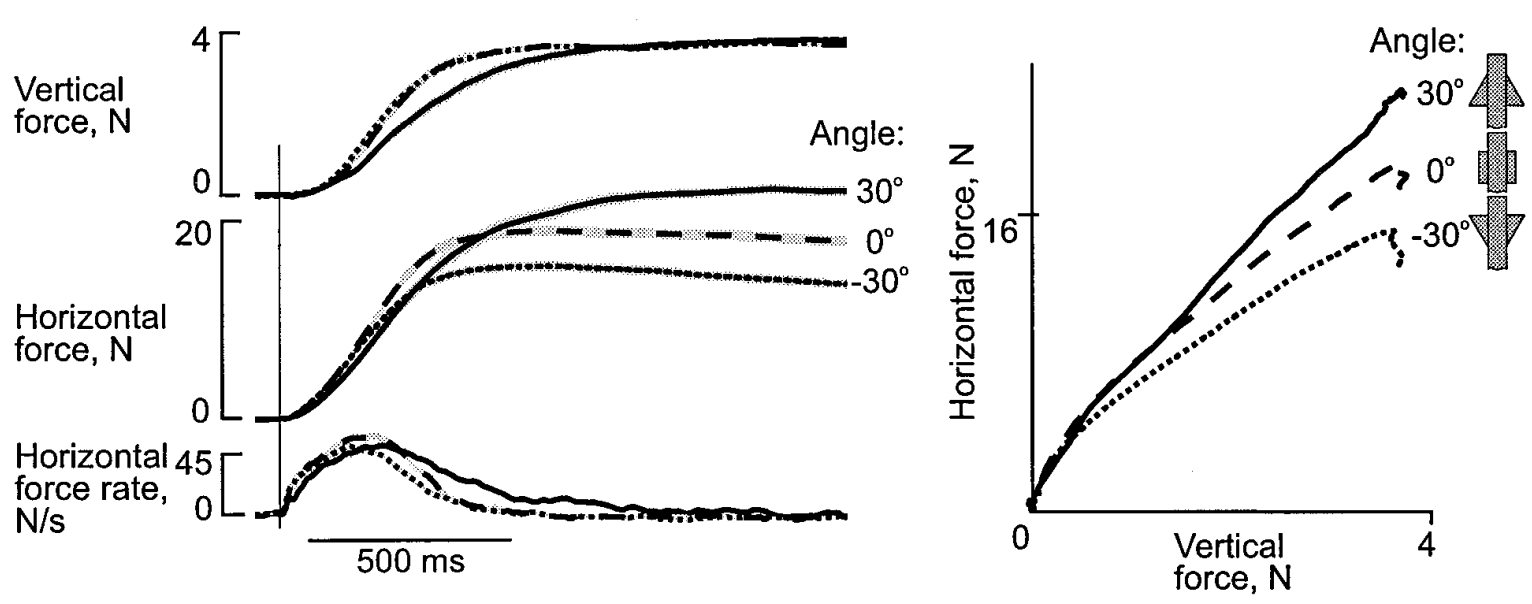

Figure 9. Adaptation to shapes of objects during digital anesthesia. $A$, B, Trials with and without vision, respectively. Left panels, vertical force and horizontal forces and horizontal force rate as a function of time for trials with $30^{\circ}$ (solid lines), $0^{\circ}$ (dashed lines), and $-30^{\circ}$ (dotted lines) surface angles. Right panels, horizontal force plotted against vertical force for the same data. In addition to surface angle given in degrees, object shapes are illustrated by the shaded inset figures (compare Fig. $1 A$ ). Data are averaged across all trials by the four anesthetized subjects; trials were synchronized at the start of a horizontal force increase when the horizontal force rate exceeded $2 \mathrm{~N} / \mathrm{s}$. The shaded zones of the curves give \pm 1 SEM, and vertical lines indicate the start of horizontal force increase.

\section{DISCUSSION}

We have demonstrated that object shape strongly influenced the coordination of fingertip forces during the dynamic and static phases of a precision grip lifting task. All subjects efficiently adapted the balance between the horizontally oriented grip force and the vertical lifting force to the surface angle and thereby achieved a nearly constant ratio between the normal and tangential forces at the grasp surfaces, regardless of surface angle. Thus, just as when subjects lift, hold, and further manipulate objects with parallel vertical grip surfaces, the forces normal and tangential to the grasp surfaces increase and decrease in parallel with an approximately constant force ratio (Johansson and Westling, 1984a; Flanagan and Wing, 1993, 1995; Flanagan and Tresilian, 1994; Kinoshita et al., 1996). Despite the great variation in finger force requirements imposed by the shape changes, the force coordination used resulted in a remarkably stable force safety margin against frictional slips; i.e., the normal force used in excess of that required to prevent slippage when holding the object in air was nearly constant across the range of surface angles. However, the safety margin could vary between subjects in an idiosyncratic manner as observed previously (Westling and Johansson, 1984).

Both visual and somatosensory input could independently support the adaptation of the force output to the shape of a manipulated object. Previous studies of manipulation tasks have shown that the adaptation of fingertip forces to physical properties of objects involves subtle interplay between two control policies termed anticipatory parameter control and discrete event, sensorydriven control (Johansson, 1996). The present results are also compatible with the operation of these control principles in the case of adaptation to object shape, as will be detailed below. Anticipatory parameter control is used to specify motor commands parametrically in advance of the movement based on previous experience with the object or estimated from perceptual cues. For example, in lifting, the development of forces immediately after the object is grasped reflects both the weight of the object and the frictional conditions in the preceding lift (Johansson and Westling, 1984a, 1988a; Edin et al., 1992; Forssberg et al., 1995). The discrete event, sensory-driven control policy uses so- 
matosensory mechanisms that sense discrete mechanical events in the digit-object interface and monitor task progress during the actual manipulation. This information is used to inform the CNS about completion of the goal for each of the subsequent action phases of the task and for triggering commands for the sequential phases of the task. Moreover, disturbances in task execution caused by erroneous anticipatory settings of the motor commands are reflected by discrete mechanical events that occur while not expected or, alternatively, that do not occur while expected. Information about mismatches between the actual sensory input and the expected input are used to trigger preprogrammed patterns of corrective responses and to update the relevant sensorimotor memories used in anticipatory parameter control. That is, a set of predicted afferent signals are considered to be generated by the neural controller in conjunction with the efferent signals and are compared with the actual afferent signals (cf. Baev and Shimansky, 1992; Merfeld et al., 1993; Miall et al., 1993; Prochazka, 1993; Abbott and Blum, 1996).

\section{Vision in anticipatory parameter control of force coordination for shapes of objects}

When we reach out to take an object we automatically use visual cues to select an appropriate grasp configuration, preshape our hand to the size and shape of the object, and place our digits onto it in a manner that promotes grasp stability during the forthcoming manipulative actions (for references, see the introductory remarks). Our results demonstrate that humans also use visual cues to adapt the force coordination parametrically to object shape in anticipation of the force requirements imposed once the object is contacted; with vision (and normal digital sensibility), object shape controlled the force output from the initial application of forces, i.e., before somatosensory information could have influenced the force output. Likewise, in the absence of somatosensory information from the digits, all sighted subjects adjusted the coordination between the horizontal and vertical forces to object shape.

We have previously demonstrated that visual information plays a role in anticipatory parameter control of fingertip forces concerning their adaptation to an object's weight. First, when humans handle common objects, the force development in the dynamic phase of the lifting action is appropriately scaled for the weight of the current object despite different weights, sizes, and densities of such objects (Gordon et al., 1993). That is, memories from previous manipulative experiences are used to scale the force output to an expected weight before explicit sensory information about the weight is available at liftoff. The underlying process involves visual identification of the target object and the retrieval of implicit memory information of its physical properties in terms of the forces to apply. Second, humans can parameterize the forces in anticipation of the weight of an object from implicit knowledge about size-weight relationships of classes of related objects (Gordon et al., 1991). The present results indicate a similar ability to associate the shape of an object and the required force coordination. Relying on visual shape cues, subjects adequately anticipated the balance required between the horizontal grip force and vertical lifting force for grasp stability. Importantly, the required force coordination was directly computed and applied without previous manipulative experience of the shape of the test object. Thus, the subjects seem to have had implicit knowledge about the relationship between shape and mechanical constraints regarding force coordination required for grasp stability.

The importance of continuously seeing the object and perhaps the hand during the reach before object contact remains to be investigated. However, the fact that visual information about object shape operated parametrically on the relationship between the horizontal and vertical forces by changing its gain suggests that the ratio between these forces was determined by a force coordination memory reminiscent of that underlying anticipatory parameter control for friction between the digits and the object (Johansson and Westling, 1984a). Indeed, in daily activities we often gaze at and attend to an object while preparing to reach toward and grasp it, but when the plan is executed we often attend to stimuli in locations that differ from the target of action. Moreover, it has been demonstrated repeatedly that the kinematics of neither the reach nor the shaping of the hand are dramatically affected if vision is occluded while we reach out to grasp objects (Jeannerod, 1981, 1984; Jakobson and Goodale, 1991; Goodale et al., 1994a; Servos and Goodale, 1994; Jackson et al., 1995; Gentilucci et al., 1996). Hence, the kinematics of these movements as well as the adaptation of force coordination to object shape may be determined largely by the initial view of the object before the movement onset according to an anticipatory parameter control policy (Johansson, 1996). Premotor cortex may play an important role in these memory-based sensorimotor transformations (Wise et al., 1996). However, visual information can certainly be used to trigger corrections of reaching movements when an the location or size of an object is perturbed (Paulignan et al., 1991a,b). This type of correction may be mediated by a control policy similar to the discrete event, sensory-driven control policy operating during actual manipulation.

\section{Somatosensory updating of force coordination parameters for object shape}

The fact that subjects adjusted the force coordination to object shape even while blindfolded demonstrated that they could use somatosensory input for this purpose, independent of vision. From a control point of view the changes in object shape were treated similarly to a change in friction while people handle objects with vertical and parallel grip surfaces. That is, subjects adjusted the balance between the horizontal and vertical forces as if the surface material was more or less slippery for objects that were tapered upward and downward, respectively (cf. Johansson and Westling, 1984a; Flanagan and Wing, 1995). Hence, the coupling between horizontal and vertical forces that has previously been described for a variety of grip tasks (see the introductory remarks for references) may represent a coordinative constraint that the neural controller exploits to support grasp stability in the presence of shape variations.

The fingertip forces reflected predictions based on sensorimotor memory related to the shape of an object in a previous lifting trial during initial contact with the object in the blindfolded condition. This also applies to lift series in which the friction between the digits and the test object is varied between trials (Johansson and Westling, 1984a; Edin et al., 1992). If there was a mismatch between the anticipated force requirements and those actually imposed by the prevailing surface angle, an adjustment of the force output was initiated some $0.1 \mathrm{sec}$ after the object was contacted. This adaptation to a new object shape mediated by somatosensory information is reminiscent of the adaptation of force coordination to frictional changes when force output changes after $\sim 0.1-0.2 \mathrm{sec}$ after the contact with the object (Johansson and Westling, 1984a; Johansson and Westling, 1987; Edin et al., 1992). This similarity in adjustments of force coordination to changes in object shape and friction suggests that a 
similar discrete event, sensory-driven control policy is used in both instances. In lifting tasks with frictional changes between trials, force coordination memory is updated by signals in tactile afferents during the initial touch and sometime later by tactile afferent responses to slip (Johansson and Westling, 1984a; Johansson and Westling, 1987).

However, in contrast to object shape, available data suggest that vision is of little importance for anticipatory adjustments of the force output to frictional conditions (Edin et al., 1992). Rather, signals in tactile afferents innervating the object-digit interface seem to be used exclusively in frictional adaptation (Johansson and Westling, 1987) in combination with anticipatory parameter control based on force coordination requirements in previous lifts.

Interestingly, only with blindfolded subjects did the surface angle in the previous trial influence force coordination throughout the increase in finger force. That is, even after the force coordination had been initially updated to the change in shape, signs of the previous coordination still remained. This type of incomplete updating also occurs after frictional changes (Johansson and Westling, 1984a). Nevertheless, the effects of the actual friction or shape are considerably stronger than those of the previous trial.

There were some further differences in the coordination between the horizontal force and vertical force during the vision and no vision conditions. Blindfolded subjects used higher horizontal forces, particularly in trials in which the force requirement was not so great $\left(-30^{\circ}\right.$ and $\left.0^{\circ}\right)$; i.e., they used a larger safety margin against frictional slips in these trials. Interestingly, if visual feedback is not available, subjects also program a larger margin of error while reaching to grasp objects by increasing their grasp aperture compared with sighted conditions (Wing et al., 1986; Jakobson and Goodale, 1991; Chieffi and Gentilucci, 1993).

\section{Somatosensory afferent sources}

The experiments with blindfolded subjects whose digital nerves were anesthetized revealed that signals from receptors proximal to the interphalangeal joints were insufficient for mediating an appropriate adaptation of the fingertip forces to object shape. Indeed, joint and muscle receptors are surprisingly insensitive to events in the digit-object interfaces during grip tasks (Macefield and Johansson, 1996) and are insufficient to mediate sound reactive control of grasp stability (Johansson et al., 1992; Häger-Ross and Johansson, 1996). Likewise, during digital nerve block or topical anesthesia of the fingertips, subjects show an impaired adaptation of force coordination to the frictional condition in the digit-object interfaces (Johansson and Westling, 1984a; Edin et al., 1992).

While adjusting force coordination to object shape, it is likely that blindfolded subjects primarily used signals in populations of tactile afferents that have a receptive field in the contact area. These afferents offer many types of information that may be of relevance for the control of fingertip forces in manipulation, for instance, information related to frictional slips and creep (Johansson and Westling, 1987; Srinivasan et al., 1990; Milner et al., 1991), the shape of the contact surface (Goodwin et al., 1995), and contact angle (Goodwin and Morley, 1987), as well as distribution within the contact area of normal and tangential forces (Johansson and Westling, 1987; Srinivasan et al., 1990; Macefield et al., 1996). Thus, information related to object shape in the present study should have been readily available from signals in populations of tactile afferents. However, because blindfolded subjects still showed some adjustments to the object shape with digital nerve blocking, we cannot exclude that afferent input from sensors proximal to digits could provide some shape cues, although with considerably less fidelity than the cutaneous afferents (cf. HägerRoss and Johansson, 1996).

Finally, we conclude that in goal-directed grasping and manipulation, object shape is one factor that influences fingertip forces. Grasp stability depends on automatic sensory control in which predictive feed-forward mechanisms use somatosensory and visual signals with sensorimotor memory systems. Memory representations of relevant physical properties of the task play a pivotal role, and anticipatory strategies are crucial when purposeful actions arise from learned relationships between sensory signals and efferent commands.

\section{REFERENCES}

Abbott LF, Blum KI (1996) Functional significance of long-term potentiation for sequence learning and prediction. Cereb Cortex 6:406-416.

Baev KV, Shimansky YP (1992) Principles of organization of neural systems controlling automatic movements in animals. Prog Neurobiol 39:45-112.

Blake A (1992) Computational modelling of hand-eye coordination. Philos Trans R Soc Lond [Biol] 337:351-360.

Cadoret G, Smith AM (1996) Friction, not texture, dictates grip forces used during object manipulation. J Neurophysiol 75:1963-1969.

Chieffi S, Gentilucci M (1993) Coordination between the transport and the grasp components during prehension movements. Exp Brain Res 94:471-477.

Cole KJ, Johansson RS (1993) Friction in the digit-object interface scales the sensorimotor transformation for grip responses to pulling loads. Exp Brain Res 95:523-532.

Desmurget M, Prablanc C, Arzi M, Rossetti Y, Paulignan Y, Urquizar C (1996) Integrated control of hand transport and orientation during prehension movements. Exp Brain Res 110:265-278.

Edin BB, Westling G, Johansson RS (1992) Independent control of fingertip forces at individual digits during precision lifting in humans. J Physiol (Lond) 450:547-564.

Flanagan JR, Tresilian JR (1994) Grip load force coupling: a general control strategy for transporting objects. J Exp Psychol Hum Percept Perform 20:944-957.

Flanagan JR, Wing AM (1993) Modulation of grip force with load force during point-to-point arm movements. Exp Brain Res 95:131-143.

Flanagan JR, Wing AM (1995) The stability of precision grip forces during cyclic arm movements with a hand-held load. Exp Brain Res 105:455-464.

Flanagan JR, Wing AM, Allison S, Spenceley A (1995) Effects of surface texture on weight perception when lifting objects with a precision grip. Percept Psychophysics 57:282-290.

Forssberg H, Eliasson AC, Kinoshita H, Westling G, Johansson RS (1995) Development of human precision grip IV. Tactile adaptation of isometric finger forces to the frictional condition. Exp Brain Res 104:323-330.

Gentilucci M, Daprati E, Gangitano M, Saetti MC, Toni I (1996) On orienting the hand to reach and grasp an object. NeuroReport 7:589-592.

Goodale MA, Jakobson LS, Keillor JM (1994a) Differences in the visual control of pantomimed and natural grasping movements. Neuropsychologia 32:1159-1178.

Goodale MA, Meenan JP, Bulthoff HH, Nicolle DA, Murphy KJ, Racicot CI (1994b) Separate neural pathways for the visual analysis of object shape in perception and prehension. Curr Biol 1:604-610.

Goodwin AW, Morley JW (1987) Sinusoidal movement of a grating across the monkey's fingerpad: effect of contact angle and force of the grating on afferent fiber responses. J Neurosci 7:2192-2202.

Goodwin AW, Browning AS, Wheat HE (1995) Representation of curved surfaces in responses of mechanoreceptive afferent fibers innervating the monkeys fingerpad. J Neurosci 15:798-810.

Gordon AM, Forssberg H, Johansson RS, Westling G (1991) Integration of sensory information during the programming of precision grip: comments on the contributions of size cues. Exp Brain Res 85:226-229.

Gordon AM, Westling G, Cole KJ, Johansson RS (1993) Memory representations underlying motor commands used during manipulation of common and novel objects. J Neurophysiol 69:1789-1796.

Häger-Ross C, Johansson RS (1996) Non-digital afferent input in reac- 
tive control of fingertip forces during precision grip. Exp Brain Res 110:131-141.

Jackson SR, Jackson GM, Rosicky J (1995) Are non-relevant objects represented in working memory? The effect of nontarget objects on reach and grasp kinematics. Exp Brain Res 102:519-530.

Jakobson LS, Goodale MA (1991) Factors affecting higher-order movement planning - a kinematic analysis of human prehension. Exp Brain Res 86:199-208.

Jeannerod M (1981) Intersegmental coordination during reaching at natural visual objects. In: Attention and performance (Long J, Baddeley A, eds), pp 153-168. Hillsdale, NJ: Erlbaum.

Jeannerod M (1984) The timing of natural prehension movements. J Mot Behav 16:235-254

Johansson RS (1996) Sensory control of dextrous manipulation in humans. In: Hand and brain: the neurophysiology and psychology of hand movements (Wing AM, Haggard P, Flanagan JR, eds), pp 381-414. San Diego: Academic.

Johansson RS, Westling G (1984a) Roles of glabrous skin receptors and sensorimotor memory in automatic control of precision grip when lifting rougher or more slippery objects. Exp Brain Res 56:550-564.

Johansson RS, Westling G (1984b) Influences of cutaneous sensory input on the motor coordination during precision manipulation. In: Somatosensory mechanisms (von Euler C, Franzen O, Lindblom U, Ottoson D, eds), pp 249-260. London: Macmillan.

Johansson RS, Westling G (1987) Signals in tactile afferents from the fingers eliciting adaptive motor responses during precision grip. Exp Brain Res 66:141-154.

Johansson RS, Westling G (1988a) Coordinated isometric muscle commands adequately and erroneously programmed for the weight during lifting task with precision grip. Exp Brain Res 71:59-71.

Johansson RS, Westling G (1988b) Programmed and triggered actions to rapid load changes during precision grip. Exp Brain Res 71:72-86.

Johansson RS, Vallbo ÅB, Westling G (1980) Thresholds of mechanosensitive afferents in the human hand as measured with von Frey hairs. Brain Res 184:343-351.

Johansson RS, Häger C, Bäckström L (1992) Somatosensory control of precision grip during unpredictable pulling loads. III. Impairments during digital anesthesia. Exp Brain Res 89:204-213.

Kelso JAS, Buchanan JJ, Murata T (1994) Multifunctionality and switching in the coordination dynamics of reaching and grasping. Hum Mov Sci 13:63-94.

Kinoshita H, Kawai S, Ikuta K, Teraoka T (1996) Individual finger forces acting on a grasped object during shaking actions. Ergonomics 39:243-256.

Macefield VG, Johansson RS (1996) Control of grip force during restraint of an object held between finger and thumb: responses of muscle and joint afferents from the digits. Exp Brain Res 108:172-184.

Macefield VG, Häger-Ross C, Johansson RS (1996) Control of grip force during restraint of an object held between finger and thumb: responses of cutaneous afferents from the digits. Exp Brain Res 108:155-171.

Merfeld DM, Young LR, Oman CM, Shelhamer MJ (1993) A multidimensional model of the effect of gravity on the spatial orientation of the monkey. J Vestib Res 3:141-161.

Miall RC, Weir DJ, Wolpert DM, Stein JF (1993) Is the cerebellum a Smith predictor? J Mot Behav 25:203-216.

Milner TE, Dugas C, Picard N, Smith AM (1991) Cutaneous afferent activity in the median nerve during grasping in the primate. Brain Res 548:228-241.

Paulignan Y, Mackenzie C, Marteniuk R, Jeannerod M (1991a) Selective perturbation of visual input during prehension movements. 1. The effects of changing object position. Exp Brain Res 83:502-512.

Paulignan Y, Jeannerod M, Mackenzie C, Marteniuk R (1991b) Selective perturbation of visual input during prehension movements. 2. The effects of changing object size. Exp Brain Res 87:407-420.

Prochazka A (1993) Comparison of natural and artificial control of movement. IEEE Trans Rehabil Eng 1:7-17.

Rosenbaum DA, Jorgensen MJ (1992) Planning macroscopic aspects of manual control. Hum Mov Sci 11:61-69.

Servos P, Goodale MA (1994) Binocular vision and the online control of human prehension. Exp Brain Res 98:119-127.

Smith AM, Scott SH (1996) Subjective scaling of smooth surface friction. J Neurophysiol 75:1957-1962.

Srinivasan MA, Whitehouse JM, LaMotte RH (1990) Tactile detection of slip: surface microgeometry and peripheral neural codes. J Neurophysiol 63:1323-1332.

Westling G, Johansson RS (1984) Factors influencing the force control during precision grip. Exp Brain Res 53:277-284.

Westling G, Johansson RS (1987) Responses in glabrous skin mechanoreceptors during precision grip in humans. Exp Brain Res 66:128-140.

Wing A, Turton A, Fraser C (1986) Grasp size and accuracy of approach in reaching. J Mot Behav 18:245-260.

Wise SP, Dipellegrino G, Boussaoud D (1996) The premotor cortex and nonstandard sensorimotor mapping. Can J Physiol Pharmacol 74:469482. 